\title{
GASTROSTOMIA ENDOSCÓPICA EM PACIENTES COM CARDIOPATIA COMPLICADA
}

\author{
Celso CUKIER, Carlos Daniel MAGNONI, Paula POLETTI e Mounib TACLA
}

RESUMO - Pacientes cardiopatas complicados com isquemia cerebral e insuficiência cardiaca necessitam terapia nutricional enteral prolongada por se apresentarem em situação de risco nutricional. Complicações advindas do uso prolongado da sonda nasoenteral poderiam ser evitadas com a execução da gastrostomia endoscópica. Esta é alternativa técnica para acesso ao tubo digestivo em nutrição enteral. O objetivo deste estudo foi avaliar o tempo de indicação para gastrostomia endoscópica em pacientes com nutrição enteral com uso de sonda nasoenteral e as principais complicações do procedimento. Doze pacientes foram submetidos a gastrostomia endoscópica, sendo oito do sexo masculino. A idade média foi de 62,42 + 22,10 anos. A indicação principal foi síndrome isquêmica cerebral após parada cardiorespiratória por patologia clínica (sete pacientes) ou cirurgia cardiovascular complicada (cinco). O tempo médio para indicação da gastrostomia endoscópica foi de 35,58 + 26,79 dias após introdução da nutrição enteral. Não houve intercorrências com o procedimento e, no periodo pós-operatório tardio, ocorreu infecção do orifício de inserção da gastrostomia endoscópica em um caso, resolvida com cuidados locais. Em conclusão, a gastrostomia endoscópica é técnica segura, com baixa incidência de complicações. Pode ser realizada em ambulatório, no leito do paciente, ou em centro de terapia intensiva e sua indicação deveria ser mais precoce.

DESCRITORES - Gastrostomia. Nutrição enteral. Cardiopatia.

\section{INTRODUÇÃO}

Pacientes cardiopatas podem desenvolver complicações cardíacas e sistêmicas com conseqüente repercussão negativa sobre o estado nutricional. Entre as cardiopatias com potencial de desnutrição encontram-se as síndromes relacionadas à insuficiência cardíaca congestiva, pós-operatório complicado de cirurgia cardíaca, patologias congênitas (transposição dos grandes vasos, comunicação interatrial, comunicação interventricular) limitação crônica ao fluxo aéreo, entre outras. Como complicação, pacientes cardiopatas desenvolvem inanição relacionada à caquexia da insuficiência cardíaca e alterações vasculares cerebrais isquêmicas e hemorrágicas.
Essas situações serão de indicação precisa de tratamento nutricional enteral ou parenteral.

Estabelecida a necessidade de terapia nutricional e possibilidade do uso da nutrição enteral, deve-se decidir pela via de acesso ao tubo digestivo. Inicialmente, opta-se pelo uso da sonda enteral locada em posição pré ou pós-pilórica, dependendo das condições do paciente e objetivos da terapia nutricional. O uso prolongado da sonda enteral, entretanto, pode proporcionar complicações mecânicas como erosão nasal e necrose, abscesso septonasal, sinusite aguda, rouquidão, otite, faringite, esofagite, ulceração e estenose esofágica, fístula traqueoesofágica, ruptura de varizes esofágicas, obstrução da sonda, saída ou migração acidental da mesma ${ }^{(5)}$.

IMEN - Instituto de Metabolismo e Nutrição. Serviço de Gastrostomia Endoscópica do Hospital do Coração, São Paulo, SP. Endereço para correspondência: Dr. Carlos Daniel Magnoni - Rua Inhambu, 917 - 04520-013 - São Paulo, SP. 
$\mathrm{Na}$ última década, desenvolveu-se a gastrostomia por via endoscópica (GEP), procedimento este comparado à gastrostomia cirúrgica, provando sua segurança e desviando a necessidade de anestesia geral e laparotomia. Possui características próprias em termos de cuidados e complicações ${ }^{(19,25,27)}$.

\section{MATERIAL E MÉTODO}

Foram realizadas gastrostomias endoscópicas em 12 pacientes, oito do sexo masculino $(62,42+22,10$ anos). A principal indicação foi acidente vascular cerebral isquêmico após parada cardiorespiratória e cirurgia cardiovascular, o que ocorreu em 12 pacientes. A sonda utilizada foi a gástrica universal Freka-PEG-Systems Fr. 15 (Fresênius Laboratórios LTDA - Campinas, SP, Brasil), com 35 centímetros de comprimento.

A técnica adotada foi a descrita por GAUDERER et al. ${ }^{(11)}$. Após a introdução do endoscópio, procede-se à transiluminação da câmara gástrica para a pele. No local da localização luminosa do estômago, após anestesia local, é inserida agulha e, por meio desta, passado fio guia, que é resgatado pelo endoscópio com pinça de biopsia e é retirado do sistema digestório. A sonda enteral é laceada no fio guia que, por meio de tração, é reintroduzido, levando-a para a câmara gástrica, exteriorizando-a. Segue-se a introdução do endoscópio para a averiguação do local de inserção da sonda. Sua fixação externa dá-se por meio de anel próprio, não necessitando de pontos.

Foram considerados o tempo médio para indicação da GEP, dificuldades e complicações intra-operatórias, tempo para reinício da dieta enteral e complicações pós-operatórias (Tabela 1).
Relato de esofagite ocorreu durante a realização do procedimento, provavelmente devida à permanência prolongada da sonda nasoenteral.

\section{RESULTADOS}

O tempo médio para indicação da GEP foi de 35,38 + 26,79 dias após introdução da nutrição enteral. Todos os procedimentos ocorreram sem intercorrências e foram concluídos. As dificuldades encontradas basearam-se na identificação da câmara gástrica. Um dos pacientes apresentava estômago intra-torácico e outro "situs inversus totalis", causando aumento no tempo cirúrgico de identificação do local para punção gástrica. A dieta enteral foi reiniciada após 6 a 8 horas do procedimento com $50 \%$ do volume estimado e o volume total foi atingido após 36 horas. No período pós-operatório, ocorreu infecção no local da inserção da sonda em um caso, que foi resolvida com limpeza local e curativos, sem a necessidade de antibióticos.

\section{DISCUSSÃO}

Acesso ao sistema digestório por meio de sonda nasoenteral é fato comum no âmbito hospitalar. Sua incidência é variada de acordo com o serviço médico, na dependência do tipo de paciente e instituição. Em enfermaria geriátrica esta prática ocorreu em $6 \%$ no período de 1 ano em que 2.332 pacientes idosos foram internados ${ }^{(9)}$. A presença da sonda nasoenteral por tempo prolongado pode, entretanto, ocasionar complicações como migração ou retirada espontânea, lesões isquêmicas do nariz, orofaringe e esôfago e esofagite de refluxo, com posterior estenose esofágica, que contribuirão de forma desfavorável na evolução

TABELA 1 - Tempo médio para indicação da GEP, causas primárias das indicações e intercorrências

\begin{tabular}{|c|c|c|c|c|c|c|}
\hline Nome & $\begin{array}{c}\text { Tempo de } \\
\text { indicação } \\
\text { (dias) }\end{array}$ & $\begin{array}{c}\text { Idade } \\
\text { (anos) }\end{array}$ & Sexo & $\begin{array}{l}\text { Causa } \\
\text { Primária }\end{array}$ & Indicação & Intercorrências \\
\hline $\mathrm{JMF}$ & 30 & 63 & M & $\mathrm{MCP}$ & SIC & Estômago intra-abdominal \\
\hline FCS & 13 & 72 & M & AVCI & SIC & \\
\hline LHV & 30 & 83 & $\mathrm{~F}$ & $\mathrm{MCP}$ & $\mathrm{SIC}$ & \\
\hline EG58 & 74 & $\mathrm{~F}$ & $\mathrm{PO}$ & $\mathrm{SIC}$ & & \\
\hline LJH & 89 & 65 & M & $\mathrm{PO}$ & $\mathrm{SIC}$ & Fratura da sonda na inserção do equipo \\
\hline $\mathrm{MZC}$ & 45 & 32 & M & PCR & SIC & \\
\hline MPSN & 73 & 8 & M & $\mathrm{PO}$ & SIC & Situs inversus, esofagite, infecção orifício \\
\hline $\mathrm{BB}$ & 4 & 88 & $\mathrm{~F}$ & $\mathrm{MCP}$ & $\mathrm{SIC}$ & \\
\hline $\mathrm{AH}$ & 37 & 69 & M & $\mathrm{PO}$ & $\mathrm{SIC}$ & Esofagite \\
\hline ASO & 30 & 45 & M & $\mathrm{PO}$ & SIC & \\
\hline LP & 13 & 73 & M & $\mathrm{MCP}$ & $\mathrm{SIC}$ & \\
\hline MWMI & 5 & 77 & $\mathrm{~F}$ & AVCI & SIC & Gastrite antral \\
\hline Média & 35,58 & 62,42 & & & & \\
\hline Desvio padrão & 26,79 & 22,10 & & & & \\
\hline
\end{tabular}

PO: Pós-operatório de cirurgia cardíaca com circulação extracorpórea; MCP: Miocardiopatia complicada;

AVCI: Acidente vascular cerebral isquêmico; PCR: Parada cardiorespiratória; SIC: Síndrome de insuficiência cardíaca. 
clínica do paciente ${ }^{(14)}$. Estas intercorrências poderiam ser evitadas utilizando-se da gastrostomia que permite a manutenção da nutrição enteral sem a presença da sonda nasoenteral.

A gastrostomia é procedimento adotado em pacientes com nutrição enteral prolongada (NEP) como alternativa ao uso da sonda nasoenteral. Pode ser efetuada de forma cirúrgica aberta, laparoscópica ou endoscópica.

A GEP oferece vantagens sobre as técnicas cirúrgicas abertas por ser menos invasiva e ter custo menor. Proporciona custos (US\$ X $\left.10^{3}\right)$ menores que a gastrostomia pela técnica aberta $(119,8+65,1)$, já que pode ser realizada no centro cirúrgico $(122,4+75,7)$, na sala de endoscopia $(71,9+37,7)$ ou no leito do paciente $(78,2+37,4)$ em unidade de terapia intensiva, caso esteja em situação crítica ${ }^{(15)}$.

$\mathrm{O}$ acesso gástrico ou jejunal por meio deste método teve como indicação principal a NEP (97\%), seguida por descompressão gástrica ( $3 \%$ ) e neoplasia de cabeça e pescoço (12\%), onde se torna dificultoso o acesso enteral por meio de sonda nasogástrica ${ }^{(16,34)}$. Pacientes neurológicos tem a GEP indicada com freqüência e esta, além de prevenir as complicações da presença da sonda nasoenteral, proporciona facilidades no tratamento junto ao paciente e à família ${ }^{(3,7)}$. Em patologias inflamatórias como a doença de Crohn, em que o paciente apresenta síndromes de má absorção, a GEP pode ser realizada obtendo-se benefícios sobre o estado nutricional, preservando a massa corpórea magra e promovendo melhora na qualidade de vida ao paciente ${ }^{(23)}$.

No presente estudo, os pacientes submetidos a GEP eram todos cardiopatas complicados com neuropatia central, cuja terapia nutricional enteral havia sido programada para administração por período prolongado. A realização da GEP pode prevenir complicações mecânicas relacionadas à permanência da sonda enteral.

Indicações menos comuns como anorexia nervosa e hiperemese gravídica, também têm sido referidas como beneficiadas com o uso da $\mathrm{GEP}^{(12,29)}$.

A indicação de GEP tem sido precoce em determinados serviços em que atua equipe multidisciplinar de terapia nutricional, ocorrendo, por vezes, após 3 semanas a partir da introdução da nutrição enteral ${ }^{(21)}$. Determinadas situações clínicas, que sabidamente necessitarão de terapia nutricional enteral por tempo prolongado, podem ter a indicação da GEP mais precoce. SHERIDAN et al. ${ }^{(30)}$ descreveram o uso de GEP precocemente em pacientes com $38 \%$ de área queimada, facilitando a manutenção do estado nutricional e prevenindo complicações relacionadas à desnutrição neste grupo de pacientes.

No presente estudo a indicação da GEP ocorreu 35,58 + 26,79 dias após a introdução da terapia nutricional enteral. Observou-se que esta indicação poderia ter sido anterior, já que a necessidade de terapia nutricional estava indicada para longo prazo, segundo o quadro clínico dos pacientes, que exibiam baixo nível de consciência e impossibilidade de realimentação pela via oral em período breve. $\mathrm{O}$ longo tempo para indicação da GEP deveu-se principalmente à falta de conhecimento da equipe clínica em relação ao procedimento. Este fator pode ser atenuado com programas de educação continuada da equipe multiprofissional de terapia nutricional. Torna-se interessante a observação de que a GEP possibilita menor tempo de hospitalização em pacientes disfágicos se for instalada precocemente ${ }^{(1,17)}$.

No presente estudo, observou-se indicação mais precoce nas GEPs efetuadas posteriormente. Isto deveu-se, possivelmente, ao conhecimento de clínicos e cirurgiões quanto ao método realizado.

A opção do emprego da GEP torna-se atraente pelo sucesso obtido no procedimento, situando-se geralmente acima de $90 \%{ }^{(28,33)}$. Na presente série, não se observaram complicações durante o procedimento e em todos os casos o objetivo cirúrgico foi alcançado. As dificuldades estiveram situadas na localização do corpo gástrico. Em um dos casos o estômago situava-se em posição intratorácica e em outro havia dextro-versão com localização contralateral do estômago. Essas dificuldades foram facilmente contornadas com a transiluminação adequada e localização gástrica. Caso a localização do estômago não fosse adequada, o procedimento deveria ter sido abandonado.

A realimentação do paciente após a GEP pode ser iniciada após 8 horas do procedimento. Após o início, deve-se progredir gradualmente o volume dietético, até que o volume alvo seja atingido. Neste estudo, os pacientes receberam o fornecimento energético objetivado de 30 $\mathrm{kcal} / \mathrm{kg} / \mathrm{dia}$ após 36 horas do procedimento, tempo esse semelhante ao de CARRILLO et al. ${ }^{(4)}$, que forneceram o volume dietético total após 48 horas.

As complicações com a GEP devem ser conhecidas pelo médico no intuito de sua resolução. Podem ser divididas em complicações menores (inflamação do orifício de inserção, deslocamento da porção jejunal da sonda, vômitos, hematoma duodenal, pneumoperitônio sem peritonite, obstrução da sonda, desconexão do disco intragástrico e necessidade de recolocação da sonda) ou maiores (pneumonia, fístula gastrocólica, pneumoperitônio complicado, fasceíte necrotizante, perfuração gástrica e peritonite). Essas complicações ocorrem de 2\% a $12 \%$ dos casos, sendo de $12 \%$ a $22,5 \%$ para as menores e $3 \%$ a $17,5 \%$ para as maiores ${ }^{(7,8}$, 10,16,18,31,32,35). Em estudo multicêntrico reportando 1.500 GEPs, as complicações maiores ocorreram de $0 \%$ a $4,4 \%$ com mortalidade precoce (menos de 30 dias), média de $0,6 \%{ }^{(25)}$. A mortalidade relacionada à GEP encontra-se em $2 \%$ nos primeiros 30 dias $^{(17)}$. Neste trabalho não se observou mortalidade relacionada ao procedimento.

A peritonite, situação temida por sua gravidade, ocorreu em 5 de 1.338 casos reportados por MAMEL ${ }^{(24)}$. Este diagnóstico deve ser efetuado rapidamente e, se corrigido precocemente, as chances de evolução favorável estarão ampliadas.

Outras complicações existentes na GEP como aspiração pulmonar, ocorreram em $6,5 \%$ dos casos e não diferiram dos pacientes com sonda nasoenteral ${ }^{(2)}$. Menor incidência de alterações disfágicas foi observada com a GEP em relação à sonda nasoenteral (13\% x 17\%), o mesmo ocorrendo em relação a sangramento digestivo e dor abdominal $(2 \% \times 11 \%)^{(2)}$. 
A mortalidade tardia (após 30 dias) estará na dependência da população estudada e da precocidade da indicação da GEP. FINUCANE et al. ${ }^{(9)}$ observaram mortalidade em $25 \%$ dos pacientes submetidos a GEP nos primeiros 90 dias. A mortalidade esteve relacionada, principalmente, à própria patologia e não ao procedimento efetuado.

AMANN et al..$^{(1)}$ observaram que, em amostra de 227 pacientes submetidos a GEP, taxa de $17 \%$ de complicações relacionadas ao procedimento ocorreram nos pacientes com índice de massa corpórea diminuída e portadores de doença maligna. É possível que a seleção adequada de pacientes com prognóstico de sobrevida melhor modifiquem esta estatística ${ }^{(11)}$.

No intuito de diminuir a incidência de infecção pós-operatória no orifício de inserção da GEP, GOSSNER et al. ${ }^{(13)}$ preconizam a utilização de antibioticoprofilaxia. Neste estudo não se observou mortalidade relacionada ao procedimento. Em um dos casos ocorreu infecção do orifício da gastrostomia que foi resolvida com limpeza local, sem a necessidade de uso de antibióticos. Outras complicações não foram observadas até o presente momento.
A terapêutica nutricional por meio da GEP pode beneficiar outros objetivos do tratamento da doença de base. A manutenção ou ganho do peso corpóreo foi observada em pacientes com anorexia nervosa e hiperemese gravídica ${ }^{(12,29)}$, síndrome da imunodeficiência adquirida ${ }^{(6)}$ e pacientes oncológicos ${ }^{(22)}$. Nesta série, a manutenção do peso corpóreo e estabilização dos dados bioquímicos demonstraram a eficiência do procedimento no sentido de manutenção do estado nutricional dos pacientes submetidos a GEP.

Aspectos éticos devem ser considerados na realização da GEP. A precisa exposição da sua indicação e dos objetivos terapêuticos devem ser participados e discutidos pelo médico com o paciente e a sua família ${ }^{(26)}$. A técnica deve, entretanto, ser tratada com respeito e cuidados de cirurgia de porte maior, no intuito de prevenir complicações ${ }^{(18,32)}$.

Neste estudo pôde-se concluir que a GEP é método seguro e apresenta baixa incidência de complicações. Pode ser realizada em ambulatório ou no leito do paciente em terapia intensiva. Sua indicação deve ser mais precoce nos casos em que a terapia nutricional enteral seja programada para longo período, respeitando-se o estado clínico do paciente.

Cukier C, Magnoni CD, Poletti P, Tacla M. Percutaneous endoscopic gastrostomy in cardiologic complicated patients. Arq Gastroenterol 2000 37(4):208-212.

ABSTRACT - Complicated cardiologic patients with brain ischemia and heart failure need long term enteral nutrition. Long term nasoenteral tuibe feeding may cause complications that could be avoided with percutaneous endoscopic gastrostomy. The aim of this study was to evaluate the indications for percutaneous endoscopic gastrostomy and its main complications. Twelve patients were submitted to percutaneous endoscopic gastrostomy (eight male) with main age of 62,42 $\pm 22,10$ years old. Brain ischemia was the main indication of percutaneous endoscopic gastrostomy and occurred after 35,58 $\pm 26,79$ days, after iniciated enteral nutrition. There were no complications during procedure. On late post operatory period there were local infection in one cases, treated with local care. In conclusion, percutaneous endoscopic gastrostomy is a secure technique with low incidence of complications and its indication should be earlier.

HEADINGS - Gastrostomy. Enteral nutrition. Heart diseases.

\section{REFERÊNCIAS BIBLIOGRÁFICAS}

1. Amann W, Mischinger HJ, Berger A, Rosanelli G, Schweiger W, Werkgartner G, Fruhwirth J, Hauser H. Percutaneous endoscopic gastrostomy (PEG). 8 years of clinical experience in 232 patients. Surg Endosc 1997;11:741-4.

2. Baeten $\mathrm{C}$, Hoefnagels J. Feeding via nasogastric tube or percutaneous endoscopic gastrostomy. A comparision. Scand J Gastroenterol 1992; 27:95-8.

3. Britton JE, Lipscomb G, Mohr PD, Rees WD, Young AC. The use of percutaneous endoscopic gastrostomy (PEG) feeding tubes in patients with neurological disease. J Neurol 1997;244:431-4.

4. Carrillo EH, Heniford BT, Osborne DL, Spain DA, Miller FB, Richardson JD. Bedside percutaneous endoscopic gastrostomy. A safe alternative for early nutritional support in critically ill trauma patients. Surg Endosc 1997; 11:1068-71.
5. Coppini LZ, Waitzberg DL. Complicações da nutrição enteral. In: Waitzberg DL, ed. Nutrição enteral e parenteral na prática clínica. São Paulo: Atheneu; 1995. p.198-205.

6. Crotty B, McDonald J, Mijch AM, Smallwood RA. Percutaneous endoscopic gastrostomy feeding in AIDS. J Gastroenterol Hepatol 1998;13:371-5.

7. Fertl E, Steinhoff N, Schöfl R, Pötzi R, Doppelbauer A, Müller C; Auff E. Transient and long-term feeding by means of percutaneous endoscopic gastrostomy in neurological rehabilitation. Eur Neurol 1998;40:27-30.

8. Finocchiaro C, Galletti R, Rovera G, Ferrari A, Todros L, Vuolo A, Balzola F. Percutaneous endoscopic gastrostomy: a long-term follow-up. Nutrition 1997; 13:520-3.

9. Finucane P, Aslan SM, Duncan D. Percutaneous endoscopic gastrostomy in elderly patients. Postgrad Med J 1991;67:371-73. 
10. Fox VL, Abel SD, Malas S, Duggan C, Leichtner AM. Complications following percutaneous endoscopic gastrostomy and subsequent catheter replacement in children and young adults. Gastrointest Endosc 1997;45:64-71.

11. Gauderer MWL, Ponsky JL, Izant RJ. Gastrostomy without laparotomy: a percutaneous endoscopic technique. J Pediatr Surg 1980;15:872-5.

12. Godil A, Chen YK. Percutaneous endoscopic gastrostomy for nutrition support in pregnancy associated with hyperemesis gravidarum and anorexia nervosa. JPEN J Parenter Enteral Nutr 1998;22:238-41.

13. Gossner L, Keymling J, Hahn EG, Ell C. Antibiotic prophylaxis in percutaneous endoscopic gastrostomy (PEG): a prospective randomized clinical trial. Endoscopy 1999;31:119-24.

14. Hamaqui E, Kodsi R. Complications of enteral feeding and their prevention. In: Rombeau JL, Rolandelli RH, editors. Clinical nutrition. Enteral and tube feeding. Philadelphia, W.B. Saunders, 1997. p.554-74.

15. Harbrecht BG, Moraca RJ, Saul M, Courcoulas AP. Percutaneous endoscopic gastrostomy reduces total hospital costs in head-injured patients. Am J Surg 1998; 176:311-4.

16. Hoffer EK, Cosgrove JM, Levin DQ, Herskowitz MM, Sclafani SJ. Radiologic gastrojejunostomy and percutaneous endoscopic gastrostomy: a prospective, randomized comparison. J Vasc Interv Radiol 1999;10:413-20.

17. Hull MA, Rawlings J, Murray FE, Field J, McIntyre AS, Mahida, YR, Hawkey CJ, Allison SP. Audit of outcome of long-term enteral nutrition by percutaneous endoscopic gastrostomy. Lancet 1993;341:869-71.

18. Khattak IU, Kimber C, Kiely EM, Spitz L. Percutaneous endoscopic gastrostomy in paediatric practice: complications and outcome. J Pediatr Surg 1998;33:6772 .

19. Larson DE, Barton DD, Schroeder KW, Dimagno EP. Percutaneous endoscopic gastrostomy: indication, success, complications and mortality in 314 consecutive patients. Gastroenterology 1987;93:48-52.

20. Lees J. Nasogastric and percutaneous endoscopic gastrostomy feeding in head and neck cancer patients receiving radiotherapy treatment at a regional oncology unit: a two year study. Eur J Cancer Care (Engl) 1997;6:45-9.

21. Ljungstrom S, Backman L. Experience with percutaneous endoscopic gastrostomies in Sweden. Gastroenterology Nursing 1993;88:241-3.

22. Löser C, Wolters S, Fölsch UR. Enteral long-term nutrition via percutaneous endoscopic gastrostomy (PEG) in 210 patients: a four-year prospective study. Dig Dis Sci 1998;43:2549-57.
23. Mahajan L, Oliva L, Wyllie R, Fazio V, Steffen R, Kay M. The safety of gastrostomy in patients with Crohn's disease. Am J Gastroenterol 1997;92:985-8.

24. Mamel JJ. Percutaneous endoscopic gastrostomy. Am J Gasroenterol 1989;84:703-10.

25. Miller RE, Castlemain R, Lacqua FJ, Kotler DP. Percutaneous endoscopic gastrostomy. Results in 316 patients and review of literature. Surg Endosc 1989;3:186-90.

26. Rabeneck L, McCullough LB, Wray NP. Ethically justified, clinically comprehensive guidelines for percutaneous endoscopic gastrostomy tube placement. Lancet 1997;349:496-8.

27. Safadi BY, Marks JM, Ponsky JL. Percutaneous endoscopic gastrostomy. Gastrointest Endosc Clin North Am 1998;8:551-68.

28. Sangster W, Cuddington GD, Bachulis BL. Percutaneous endoscopic gastrostomy. Am J Surg 1988;155:677-9.

29. Serrano P, Velloso A, García Luna PP, Pereira JL, Fernádez Z, Ductor MJ, Castro D, Tejero J, Fraile J, Romero H. Enteral nutrition by percutaneous endoscopic gastrojejunostomy in severe hyperemesis gravidarum: a report of two cases. Clin Nutr 1998;17:135-9.

30. Sheridan R, Schulz J, Ryan C, Ackroyd F, Basha G, Tompkins R. Percutaneous endoscopic gastrostomy in burn patients. Surg Endosc 1999;13:401-2.

31. So JB, Ackroyd FW. Experience of percutaneous endoscopic gastrostomy at Massachusetts General Hospital - indications and complications. Singapore Med J 1998;39:560-3.

32. Tan W, Rajnakova A, Kum CK, Alponat A, Goh PM. Evaluation of percutaneous endoscopic gastrostomy in a university hospital. Hepatogastroenterology 1998;45:2060-3.

33. Thaicher RS, Ferguson, DR, Paradis, K. Percutaneous endoscopic gastrostomy: a preferred method of feeding tube gastrostomy. Am J Gastroenterol 1984;79:74850 .

34. Urban KG, Terris DJ. Percutaneous endoscopic gastrostomy by head and neck surgeons. Otolaryngol Head Neck Surg 1997;116:489-92.

35. Wijdicks EF, McMahon MM. Percutaneous endoscopic gastrostomy after acute stroke: complications and outcome. Cerebrovasc Dis 1999;9:109-11.

Recebido para publicação em 24/1/2000. Aprovado para publicação em 12/5/2000. 